\title{
Application du procédé séchage-friture aux amandes de karité : influence sur la composition en matières insaponifiables du beurre
}

Hilaire Macaire WOMENI

Robert NDJOUENKEU ${ }^{2}$

César KAPSEU ${ }^{2}$

Félicité Tchouanguep MBIAPO'

Michel LINDER ${ }^{3}$

Jacques $\mathrm{FANNI}^{3}$

${ }^{1}$ Département de biochimie,

Faculté des sciences, Université de Dschang,

67 Dschang, Cameroun

<womeni@yahoo.fr>

${ }^{2}$ Ecole nationale supérieure des sciences

agro-industrielles (ENSAI),

Université de Ngaoundéré, 455

Ngaoundéré, Cameroun

${ }^{3}$ Laboratoire de science et génie alimentaires

(LSGA), Nancy-Université,

2 avenue de la Forêt de Haye 172

F-54505, Vandœuvre-les-Nancy cedex, France

\begin{abstract}
The effect of deep fat frying dehydration conditions of shea kernels on the composition and properties of the unsaponifiable fraction of its butter was carried out by using a central composite experimental design. Both content and composition of the unsaponifiable matters depend on the thickness of kernels, the temperature of frying, the kernels/butter weight ratio and the time of frying. Butters with high content of unsaponifiable $(>6 \%)$ are extracted from the kernels with thickness less than $10 \mathrm{~mm}$ fried at less than $150^{\circ} \mathrm{C}$. The content of triterpenic alcohols is over $70 \%$ in low temperature frying conditions $\left(<150^{\circ} \mathrm{C}\right)$ of kernels of thickness under $10 \mathrm{~mm}$, fried during 4-8 minutes under a weight ratio lower than 0.060 . The quantity of hydrocarbons is between 4 and $57 \%$, depending on frying conditions of the kernels. The best result is obtained by frying during 2-8 minutes kernels with less than $8 \mathrm{~mm}$ of thickness at a temperature less than $150^{\circ} \mathrm{C}$ in a kernels/butter ratio of 0.035-0.060. The sterols content remains lower than $7 \%$ depending on the frying conditions by $68 \%$.
\end{abstract}

Key words: Butyrospermum parkii, shea butter, deep fat frying dehydration, unsaponifiable matterTLC-FID
Article reçu le 3 mai 2006

accepté le 5 juillet 2007

\section{FONDAMENTAI}

\section{Introduction}

Le karité, Butyrospermum parkii, est un arbre des savanes arborées soudaniennes qui donne un fruit de 40 à $50 \mathrm{~mm}$ de diamètre, à pulpe sucrée comestible couvrant une à trois graines constituées d'une amande à deux cotylédons remplissant une coque mince et rigide. L'amande est constituée de 35 à $50 \%$ de matière grasse solide à température ambiante d'où l'appellation de beurre de karité [1]. L'amande contient également un latex qui s'extrait avec le beurre dans les différents procédés d'extraction. Ce beurre a une teneur très élevée et une composition particulière en matières insaponifiables. L'insaponifiable du beurre de karité est constitué en moyenne de $65 \%$ d'alcools triterpéniques ( $\alpha$ et $\beta$-amyrine, butyrospermol, lupéol, parkéol), de $8 \%$ de stérols ( $\Delta^{7}$-stigmastérol, $\alpha$-spinastérol) et de $27 \%$ d'hydrocarbures (karitène) [2]. La principale raison de l'intérêt accordé au beurre de karité est la reconnaissance par l'industrie et le consommateur de ses propriétés intéressantes en matière de cosmétique. L'insaponifiable et le latex sont actifs sur la protection de la peau ( $\alpha$ et $\beta$-amyrine), la cicatrisation (parkéol), la désinfection (lupéol), la stimulation des cellules ( $\alpha$-spinastérol) ; la régénération des cellules $\left(\Delta^{7}\right.$-stigmastérol), la prévention des allergies au soleil (latex). Des propriétés hydratantes, anti-inflammatoires, anti-eczéma et anti-ride ont été également soulignées [3-6].

Le séchage-friture des amandes de karité est une technique de séchage rapide qui minimise la dégradation de la qualité du beurre telle que mesurée par les indices d'acide et de peroxyde [7-9]. Pourtant, ce beurre étant surtout recherché pour la particularité de sa fraction insaponifiable, la dégradation chimique de celle-ci au cours de l'extraction a fait l'objet de travaux au cours desquels il a été observé que lors du blanchiment, les alcools triterpéniques peuvent subir une isomérisation alors que les stérols peuvent se déshydrater [10]. Le séchage-friture impliquant un traitement à température élevée est susceptible de produire de telles modifications, comme la dégradation des alcools triterpéniques qui conduit aux hydrocarbures. L'objectif de ce travail est de déterminer l'effet des conditions de séchage-friture des amandes de karité sur la teneur et la composition de l'insaponifiable du beurre.

\section{Matériel et méthodes}

Les fruits de karité utilisés ont été achetés sur le marché de Ngaoundéré (Cameroun). Les caractéristiques de la matière première (fruits, amandes, beurre de karité du bain), les conditions de 
friture et la conduite du plan d'expériences composite centré ont été précisées dans un précédent article [8].

Quatre facteurs sont étudiés : l'épaisseur des amandes, $x_{1}(2$ à $18 \mathrm{~mm})$, la température du bain de friture, $x_{2}\left(120\right.$ à $\left.180^{\circ} \mathrm{C}\right)$, le rapport massique amandes/huile, $x_{3}(0,03$ à 0,07$)$ et le temps de friture, $x_{4}(0,4$ à $11,6 \mathrm{~min})$.

Les amandes frites et refroidies à température ambiante sont broyées et les beurres extraits à l'hexane avec le dispositif de Soxhlet. La séparation de la fraction insaponifiable est réalisée par saponification de 2 à $2,5 \mathrm{~g}$ de beurre de karité par traitement à reflux en présence d'une solution éthanolique d'hydroxyde de potassium, suivie de l'extraction de l'insaponifiable au moyen d'éther diéthylique [11, 12]. La détermination de la composition des fractions insaponifiables a été réalisée par chromatographie sur couche mince couplée à la détection par ionisation de flamme (TLC-FID, latroscan ${ }^{\text {TM }}$ MK-5, IATRON Laboratories Inc. Tokyo, Japon).

La fraction insaponifiable des différents beurres a été dissoute dans le chloroforme à raison de $5 \mathrm{mg}$ d'échantillon pour $1 \mathrm{~mL}$ de chloroforme. Deux $\mu \mathrm{L}$ d'une même dilution d'échantillon sont déposés par microseringue sur deux baguettes de quartz recouvertes de silice (Chromarods $\left.^{\mathrm{TM}} \mathrm{III}\right)$. Les chromarods sont élués à température ambiante dans un mélange hexane-éther diéthylique $(70: 30)$ puis séchés à $100{ }^{\circ} \mathrm{C}$ pendant 1 minute. La détection est réalisée sur latroscan MK-5. Le chromatogramme du mélange des standards a été réalisé dans les mêmes conditions. Ce mélange était constitué d'un hydrocarbure (le squalène), d'un tocophérol (le $\delta$-tocophérol), d'un alcool triterpénique (le lupéol) et de trois stérols (stigmastérol, $\beta$-sitostérol et cholestérol).

Le logiciel STATGRAPHICS Plus 3.0 a permis de construire le plan d'expériences et d'analyser la variance des coefficients de régression des équations d'expression des teneurs en insaponifiables, alcools triterpéniques, hydrocarbures et stérols. Les courbes isoréponses ont été tracées avec le logiciel STATISTICA 6.0.

\section{Résultats et discussion}

\section{Teneur en matières insaponifiables}

L'analyse des teneurs en matières insaponifiables $(\mathrm{MI})$ a généré le modèle d'équation suivant :

$$
\begin{aligned}
\% \mathrm{Ml}= & 74,429-2,259 \mathrm{e}-0,719 \mathrm{~T}-277,91 \mathrm{r}+0,731 \mathrm{t} \\
& +0,012 \mathrm{e}^{2}+0,014 \mathrm{e} . \mathrm{T}-1,039 \mathrm{e} . \mathrm{r}+0,3.10^{-4} \mathrm{e} . \mathrm{t}+0,002 \mathrm{~T}^{2} \\
& +0,131 \mathrm{~T} \cdot \mathrm{r}-0,006 \mathrm{~T} . \mathrm{t}+3302,19 \mathrm{r}^{2}-7,3683 \mathrm{r} \cdot \mathrm{t}+0,03 \mathrm{t}^{2}
\end{aligned}
$$

où e est l'épaisseur des amandes, $T$ la température de friture, $r$ le rapport massique amandes/beurre et $t$ le temps de friture.

Ce modèle explique $40 \%$ de la variation de la teneur en insaponifiables. Elle varie de 3 à $9 \%$ et n'est significativement affectée que par l'effet $d^{\prime}$ interaction de l'épaisseur des amandes et de la température de friture $(P<0,01)$ (tableau 1). Plusieurs auteurs rapportent des teneurs variant de 2 à $17 \%$ [3-4, 13-15]. Les valeurs obtenues rentrent dans I'intervalle 3,5-8,5\% correspondant au taux d'insaponifiable du karité de la variété Poissoni. Elle s'écarte de ceux des variétés Mangifolia (5 à $17 \%$ ) et Nilotica (2,5 à $3 \%$ ) [1]. Les valeurs supérieures à $6 \%$ sont obtenues, soit avec des températures de friture supérieures à $150^{\circ} \mathrm{C}$ couplées à des épaisseurs $\mathrm{d}^{\prime}$ amandes supérieures à $10 \mathrm{~mm}$, soit avec des températures de moins de $150^{\circ} \mathrm{C}$ avec des épaisseurs inférieures à $10 \mathrm{~mm}$ (figure 1).

\section{Composition de la fraction insaponifiable}

La figure 2 présente le chromatogramme obtenu au latroscan ${ }^{\mathrm{TM}}$ des différentes classes d'insaponifiables du beurre extrait à partir d'amandes de $6 \mathrm{~mm}$ d'épaisseur frites à $165^{\circ} \mathrm{C}$ dans un rapport massique amandes/beurre de 0,060 de karité pendant 3,2 minutes. Il est constitué de quatre pics d'intensité variable apparaissant aux temps de rétention 0,$15 ; 0,28 ; 0,33$ et 0,45 minute. Par comparaison au chromatogramme du mélange des standards, ces pics ont été identifiés et correspondent aux hydrocarbures (pic 1), aux alcools triterpéniques (pic 2), aux stérols (pic 3) et aux impuretés de l'insaponifiable (pic 4). Ces espèces sont donc

\begin{tabular}{|c|c|c|c|c|c|c|c|c|}
\hline \multirow[t]{2}{*}{ Sources } & \multicolumn{2}{|c|}{ Teneur en insaponifiables } & \multicolumn{2}{|c|}{ Alcools triterpéniques } & \multicolumn{2}{|c|}{ Hydrocarbures } & \multicolumn{2}{|c|}{ Stérols } \\
\hline & CR & $\mathbf{P}$ & CR & $\mathbf{P}$ & CR & $\mathbf{P}$ & CR & $\mathbf{P}$ \\
\hline Epaisseur : $x_{1}$ & $-2,2587$ & 0,9610 & 8,7248 & $0,0134^{*}$ & $-6,9843$ & $0,0049^{*}$ & $-1,0230$ & $0,0008^{*}$ \\
\hline Température : $x_{2}$ & $-0,7185$ & 0,9513 & 4,4784 & $0,0000^{*}$ & $-1,1200$ & $0,0000^{*}$ & $-0,0421$ & $0,0000^{*}$ \\
\hline Rapport : $x_{3}$ & $-277,91$ & 0,3315 & 2179,62 & 0,1403 & $-2272,78$ & 0,1539 & 145,152 & 0,8708 \\
\hline Temps : $x_{4}$ & 0,7312 & 0,1923 & 3,5800 & 0,7664 & $-4,9443$ & 0,9273 & 0,4199 & $0,0026^{*}$ \\
\hline$x_{1}^{2}$ & 0,0121 & 0,3555 & $-0,0929$ & 0,1970 & 0,0872 & 0,2912 & 0,0141 & 0,1268 \\
\hline$x_{1} x_{2}$ & 0,0138 & $0,0005^{*}$ & $-0,0645$ & $0,0023^{*}$ & 0,0488 & $0,0381^{*}$ & 0,0075 & $0,0051^{*}$ \\
\hline$x_{1} x_{3}$ & $-1,0391$ & 0,8488 & 7,2133 & 0,8085 & 4,0938 & 0,9048 & $-5,6773$ & 0,1418 \\
\hline$x_{1} x_{4}$ & $0,3.10^{-4}$ & 0,9989 & 0,3036 & $0,0066^{*}$ & $-0,2411$ & 0,0546 & $-0,0360$ & $0,0113^{*}$ \\
\hline$x_{2}^{2}$ & 0,0020 & 0,0345 & $-0,0140$ & $0,0085^{*}$ & 0,0040 & 0,4888 & $-0,35.10^{-6}$ & 0,9996 \\
\hline$x_{2} x_{3}$ & 0,1313 & 0,9280 & 1,0277 & 0,8919 & $-2,5554$ & 0,7797 & $-0,9760$ & 0,3395 \\
\hline$x_{2} x_{4}$ & $-0,0064$ & 0,3935 & $-0,0273$ & 0,3379 & 0,0283 & 0,3881 & $-0,0044$ & 0,2324 \\
\hline$x_{3}^{2}$ & 3302,19 & 0,1197 & $-27116,8$ & $0,0217^{*}$ & 29577,2 & $0,0291^{*}$ & $-178,906$ & 0,9024 \\
\hline$x_{3} x_{4}$ & $-7,3683$ & 0,3469 & 25,4743 & 0,5498 & $-30,1406$ & 0,5388 & 12,3158 & $0,0285^{*}$ \\
\hline$x_{4}^{2}$ & 0,0300 & 0,2638 & $-0,3245$ & $0,0306^{*}$ & 0,3819 & $0,0273^{*}$ & 0,0102 & 0,5830 \\
\hline Constante & 74,4286 & 1 & $-326,181$ & 1 & 156,244 & I & 8,5895 & 1 \\
\hline $\begin{array}{l}\text { Coefficient de } \\
\text { détermination (\%) }\end{array}$ & \multicolumn{2}{|c|}{40,0883} & \multicolumn{2}{|c|}{71,7211} & \multicolumn{2}{|c|}{72,9674} & \multicolumn{2}{|c|}{68,2141} \\
\hline
\end{tabular}

Tableau 1. Coefficients de l'équation de régression (CR), coefficient de détermination et valeurs de P de l'ANOVA des modèles d'expression de la teneur et de la composition de la fraction insaponifiable (teneur en alcools triterpéniques, hydrocarbures et stérols) des beurres extraits des amandes frites selon le plan d'expériences composite centré.

* valeurs de $p<0,05$, indiquant qu'il y a effet significatif de 0 à $95 \%$ niveau de confiance. 


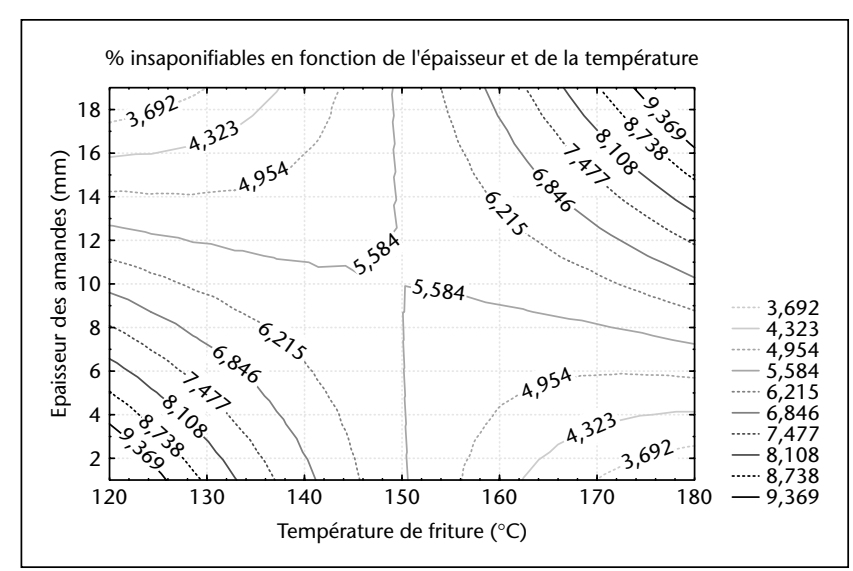

Figure 1. Courbes isoréponses montrant la variation de la teneur en matières insaponifiables en fonction de l'épaisseur des amandes et de la température de friture.

les trois principales classes de matières insaponifiables du beurre de karité.

Les alcools triterpéniques sont les constituants majoritaires et varient de 38 à $82 \%$ en fonction des conditions de séchage-friture. Cette variation est liée pour $72 \%$ à l'épaisseur des amandes, au rapport massique amandes/beurre, à la température et au temps de friture. Les effets les plus significatifs $(P<0,01)$ sont ceux des termes de premier et de second ordre de la température, des interactions de l'épaisseur des amandes et de la température de friture, de l'épaisseur des amandes et du temps de friture. Le modèle est également affecté $(P<0,05)$ par le terme de premier ordre de l'épaisseur des amandes, et les termes de second ordre du rapport massique amandes/beurre et du temps de friture (tableau 1). Les valeurs les plus élevées (70 à $82 \%$ ) sont obtenues à partir des amandes frites dans les conditions suivantes: température inférieure à $150{ }^{\circ} \mathrm{C}$, épaisseur des amandes inférieure à $10 \mathrm{~mm}$, rapport massique inférieur à 0,06 et temps de friture de 2 à 8 minutes (figure $3 A$ et $B$ ). La friture de tranches épaisses d'amandes conduit à l'exposition longue à la chaleur de produits sensibles, séquence favorable aux réactions de dégradation des alcools triterpéniques. Il a déjà été noté qu'un séjour long des amandes de karité dans une étuve favorisait la dégradation des alcools triterpéniques [16].

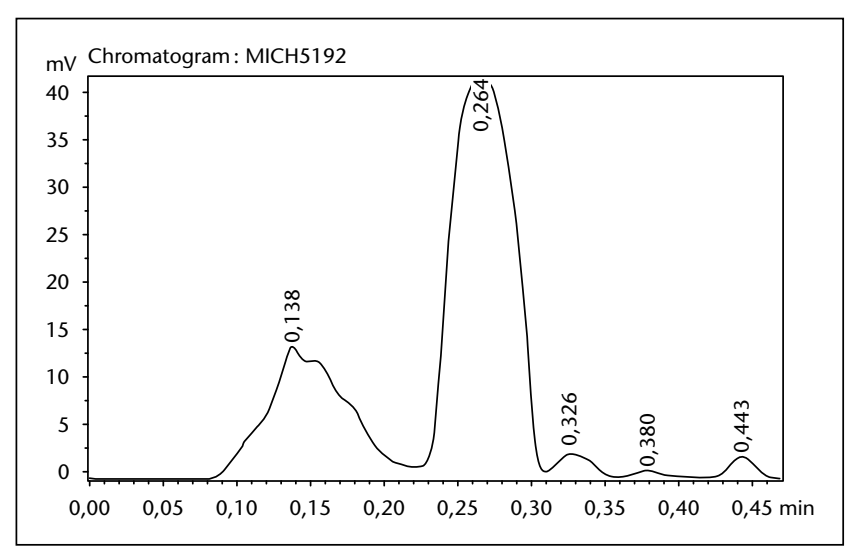

Figure 2. Chromatogramme par TLC-FID (Iatroscan ${ }^{T M}$ ) de l'insaponifiable du beurre de karité extrait à partir des amandes de $6 \mathrm{~mm}$ d'épaisseur frites à $165^{\circ} \mathrm{C}$ dans le rapport massique amandes/beurre de 0,060 de karité pendant 3,2 minutes. Système de solvant d'élution : hexane-diéthyl éther (70:30).
(A) \% alcools triterpéniques en fonction de l'épaisseur et de la température

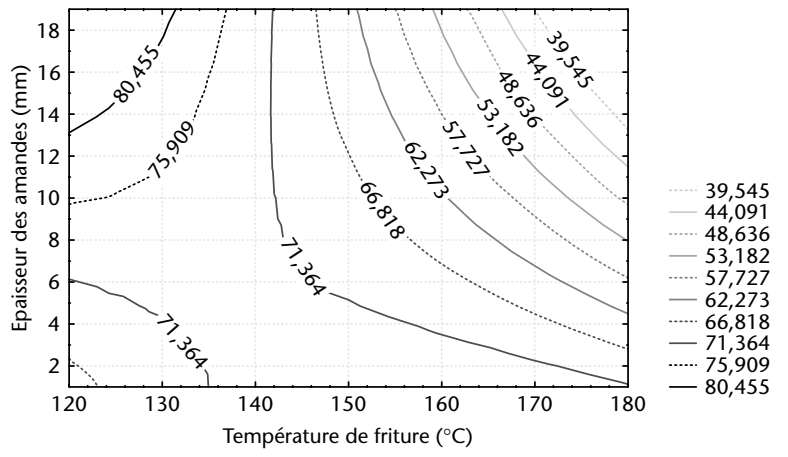

(B) \% alcools triterpéniques en fonction du rapport et du temps

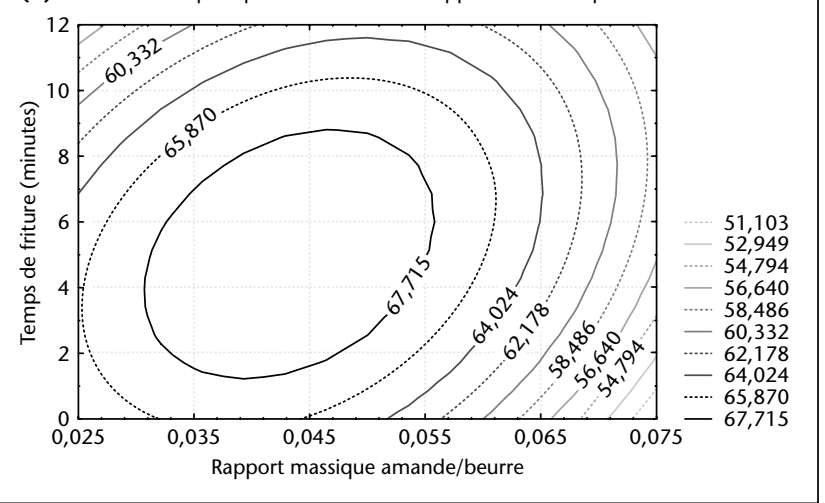

Figure 3. A) Courbes isoréponses montrant la variation de la teneur en alcools triterpéniques dans les fractions d'insaponifiables en fonction de l'épaisseur des amandes et de la température de friture. B) Courbes isoréponses montrant la variation de la teneur en alcools triterpéniques dans les fractions d'insaponifiables en fonction du rapport massique amandes/beurre et du temps de friture.

La quantité d'hydrocarbures varie entre 4 et $57 \%$. Cette variation est liée pour $73 \%$ aux conditions de friture. Les effets les plus significatifs $(P<0,01)$ sont ceux des termes de premier ordre de l'épaisseur des amandes et de la température de friture, des termes de second ordre du rapport massique et du temps de friture et enfin du terme de l'interaction entre l'épaisseur des amandes et la température de friture.

Des amandes de plus de $8 \mathrm{~mm}$ d'épaisseur frites à des températures supérieures à $150^{\circ} \mathrm{C}$ donnent des beurres dont les insaponifiables contiennent 39 à $57 \%$ d'hydrocarbures. À moins de $150^{\circ} \mathrm{C}$, la friture des amandes de même taille ou moins épaisses permet $d^{\prime}$ obtenir des insaponifiables avec 4 à $28 \%$ d'hydrocarbures (figure $4 \mathrm{~A}$ ). Les insaponifiables moins riches en hydrocarbures sont obtenus pour des durées de friture de 2 à 8 minutes réalisées avec un rapport massique de 0,035 à 0,060 (figure $4 B$ ). Pour limiter la production d'hydrocarbures, la friture devrait être conduite avec des amandes de moins de $8 \mathrm{~mm}$ d'épaisseur, dans un bain de beurre de karité porté à moins de $150^{\circ} \mathrm{C}$ dans le rapport massique amandes/beurre de 0,035-0,060 pendant 2 à 8 minutes. Ces conditions sont également celles qui ont été notées dans les paragraphes précédents comme optimales pour la préservation des alcools triterpéniques, ce qui confirme le mécanisme de dégradation. II est donc possible ainsi d'obtenir des beurres riches en alcools triterpéniques ( $\alpha$ et $\beta$-amyrine, parkéol, lupéol, butyrospermol) qui sont les principaux composés bioactifs de l'insaponifiable du beurre de karité, tout en minimisant la teneur en hydrocarbures.

Les stérols sont peu présents dans le beurre de karité : leur teneur varie de 0 à $7 \%$ et dépend à $68 \%$ des conditions de friture. Les effets les plus 


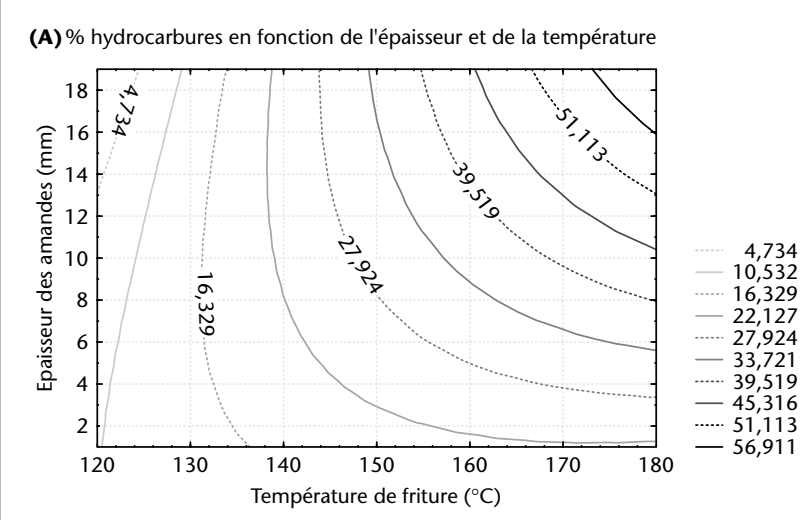

(B) \% hydrocarbures en fonction du rapport et du temps

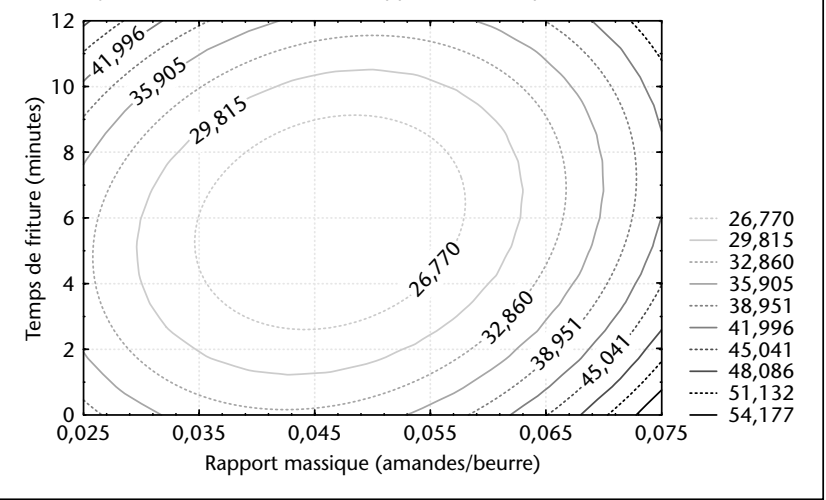

Figure 4. A) Courbes isoréponses montrant la variation de la teneur en hydrocarbures en fonction de l'épaisseur des amandes et de la température de friture. B) Courbes isoréponses montrant la variation de la teneur en hydrocarbures en fonction du rapport massique amande/beurres et du temps de friture.

significatifs $(P<0,01)$ sont ceux des termes de premier ordre de l'épaisseur des amandes, de la température de friture, du temps de friture, des termes d'interactions entre l'épaisseur des amandes et la température de friture, l'épaisseur des amandes et le temps de friture, le rapport massique et le temps de friture. Les courbes isoréponses de la figure 5 montrent que les conditions de friture favorables à la dégradation des stérols sont: températures supérieures à $150^{\circ} \mathrm{C}$ pendant moins de 4 minutes, tranches de plus de $10 \mathrm{~mm}$ d'épaisseur et rapport massique supérieur à 0,045 . Elles réduisent leur teneur à moins de $2 \%$. Ces mêmes conditions se sont révélées favorables à la production des hydrocarbures. La dégradation des stérols se ferait par déshydratation pour donner des hydrocarbures, principalement des stéradiènes. En effet, l'apparition des stéradiènes résultant de la déshydratation du $\beta$-sitostérol, du campestérol et du stigmastérol a été observée après blanchiment des huiles végétales utilisées pour la formulation des équivalents du beurre de cacao (CBE) [10]. Pour atténuer une telle dégradation des stérols, la friture des amandes de moins de $6 \mathrm{~mm}$ à moins de $150^{\circ} \mathrm{C}$ dans le rapport supérieur à 0,045 et pendant plus de 6 minutes serait adéquate. Ainsi, on préserve des composés tels que l' $\alpha$-spinastérol et le $\Delta^{7}$-stigmastérol, responsables de la stimulation et de la régénération cellulaire [3-6].

\section{Conclusion}

L'épaisseur des amandes, la température de friture et leur interaction sont les principales variables de friture qui affectent la composition de l'insa-
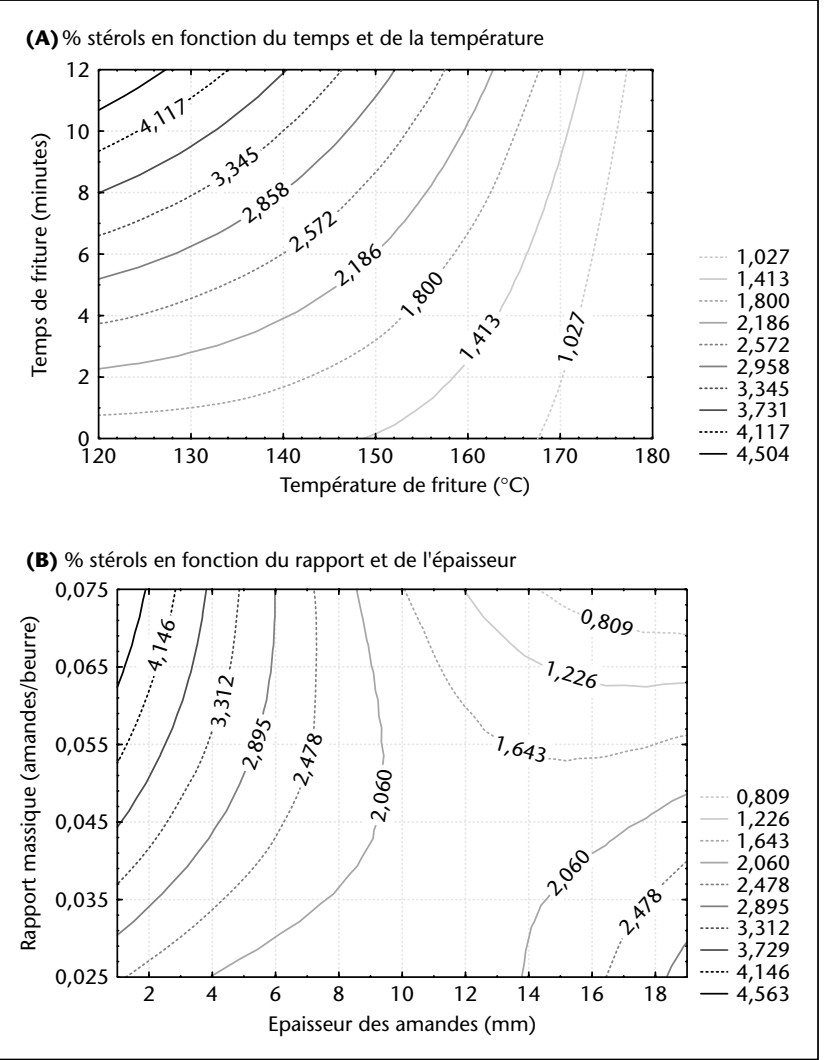

Figure 5. A) Courbes isoréponses montrant la variation de la teneur en stérols en fonction de la température et du temps de friture. B) Courbes isoréponses montrant la variation de la teneur en stérols en fonction de l'épaisseur des amandes et du rapport massique amandes/beurre.

ponifiable du beurre de karité. Les conditions qui minimisent la perte d'insaponifiable et la dégradation des alcools triterpéniques et stérols au cours du procédé séchage-friture des amandes de karité sont les suivantes: amandes de 2 à $6 \mathrm{~mm}$ d'épaisseur, température de moins de $150{ }^{\circ} \mathrm{C}$, rapport massique amandes/beurre de 0,045 à 0,060 et temps de friture de 6 à 8 minutes.

Remerciements. Les auteurs remercient la Fondation Internationale pour la Science (IFS, Stockholm, Suède) et I'Université des Nations Unies (UNU, Tokyo, Japon) pour leur support financier, ainsi que I'Institut de Recherche pour le Développement (IRD, Paris, France) qui a financé les déplacements.

\section{REFERENCES}

1. MENSIER PH. Dictionnaire des huiles végétales. Paris : Paul Chevalier, 1957; (108-10).

2. JACOBSBERG B. Causes de l'acidification du beurre du karité au cours de la préparation et du stockage des amandes. Oléagineux 1977 ; 32 : 529-33.

3. ATA JKBA. The study of the shea kernel in relation to the traditionnal process of shea fat. Ph.D. Thesis, University of Chana, Legon-Accra, 1978.

4. PESQUET JJ. Shea nuts. Aproma/Bimonthly Review $1992 ; 8$ : 27-9.

5. LAZANO YF, DHUIQUE MAYER C, BANNON C, GAYDOU EM. Unsaponifiable matter, Total Sterol and Tocopherol contents of avocado oil varieties. J Am Oil Chem Soc 1993 ; 70 : 561-5. 
6. LOVETT PN. Shea butter industry expanding in West Africa. Inform 2005 ; $16(5): 273-5$.

7. WOMENI HM. Identification et analyse des opérations critiques de préparation des fruits, graines et amandes de karité (Butyrospermum parkii (G. Don) Kotschy): étude de leur influence sur la qualité du beurre. Thèse de Doctorat/Ph. D de l'Université de Ngaoundéré, Cameroun, 2004.

8. WOMENI HM, NDJOUENKEU R, KAPSEU C, PARMENTIER M. Application du procédé séchage-friture aux amandes de karité : cinétique de séchage. $\mathrm{OCL}$ $2004 ; 11(6)$ : 457-63.

9. WOMENI HM, NDJOUENKEU R, KAPSEU C, PARMENTIER M, FANNI J. Application du procédé séchage-friture aux amandes de karité : influence sur les indices chimiques de qualité et les propriétés de fusion du beurre. OCL $2006 ; 13(4): 297-302$.

10. CREWS C, CALVET-SARRET R, BRERETON P. Identification of steroidal hydrocarbons in refined confectionery fats by gas chromatography-mass spectrometry. J Chromatogr A 1999 ; 847 : 179-85.
11. AFNOR. Recueil des normes françaises, Corps gras graines oléagineuses, produits dérivés. $5^{\mathrm{e}}$ édition. Paris : AFNOR, 1993.

12. AOAC (Association of Official Analytical Chemists). In : Patricia CUNNIFF, ed. Official Methods of Analysis, 16th édition, Volume II. Maryland : AOAC International, 1999.

13. SWERN D. Baileys Industrial Oil and Fat Products. Vol. 2, 4th edn. New York: Wiley-Interscience Publication, John Wiley \& Sons, 1979.

14. SALUNKHE DK, DESAI BB. Post-harvest Biotechnology of Oilseeds. Boca Raton: CRC Press Inc., 1986.

15. TANO-DEBRAH K, OHTA Y. Enzyme-Assisted Aqueous Extraction of Fat from Kernels of the Shea Tree, Butyrospermum parkii. / Am Oil Chem Soc 1994 ; $7(9):$ 979-83.

16. WOMENI HM, NDJOUENKEU R, KAPSEU C, TCHOUANGUEP MF, PARMENTIER M, FANNI J. XIe Conférence Annuelle de Biosciences. Cameroun : Université de Dschang, 2004. 